\title{
CAPSULE COMMENTARIES
}

\section{Capsule Commentary on Zueger et al., Older Medicare Beneficiaries Frequently Continue Medications with Limited Benefit Following Hospice Admission}

\author{
Allison Taylor, MD and Elliot $Y u, M D$ \\ Medical College of Wisconsin, Milwaukee, WI, USA. \\ $\mathrm{J}$ Gen Intern Med 34(10):2185 \\ DOI: $10.1007 / \mathrm{s} 11606-019-05262-6$ \\ (c) Society of General Internal Medicine 2019
}

$\mathrm{T}$ he transition to hospice should include a de-escalation of medications other than those that provide symptomatic relief or improve quality of life. However, the study by Zueger et al. ${ }^{1}$ reports that about one-third of patients admitted to hospice continue at least one limited benefit medication (LBM). This problematic practice carries risk of drug-drug interactions, polypharmacy, burden on caretakers, and cost although the authors acknowledge that risk-benefit of continuing LBMs is not equal across classes.

Despite risk, patients and families may hesitate before discontinuing LBMs for chronic conditions after years of counseling from providers about risk reduction. Sera et al. speculate that continuing chronic medications such as antiplatelets in patients with end-stage dementia have longer than expected clinical course when compared with hospice patients with a progressive cancer diagnosis. ${ }^{2}$ Additionally, discontinuing medicines may carry risk. There are concerns of rebound acid hyper-secretion causing dyspepsia after discontinuing proton-pump inhibitors after 8 weeks of therapy. ${ }^{3}$ Further studies investigating specific classes of LBMs are needed to provide clinical indication for discontinuation.

The study identifies factors predictive of continuing LBMs (hospice location and patients prescribed many pre-hospice LBMs) which should serve as a launchpad for quality improvement interventions for these at-risk groups. Further investigation should include the context surrounding medication reconciliation at hospice admission and the more common use of hospital-designed order sets through electronic health records.

The SEER database is a trove of data. One evocative datapoint addressed in the study is that pre-hospice medications averaged 15.7. With such significant pre-hospice medication burden, why did the authors determine outcome measurement to be continuation of just one LBM? Does the continuation of one LBM suggest continuation of other LBMs? These results could further illustrate the burden of LBMs on patients, caretakers, and healthcare system.

Corresponding Author: Allison Taylor, MD; Medical College of Wisconsin, Milwaukee, WI, USA (e-mail: actaylor@mcw.edu).

\section{Compliance with Ethical Standards:}

Conflict of Interest: The authors declare that they do not have a conflict of interest.

\section{REFERENCES}

1. Zueger P, Holmes HM, Calip GS, Gato DM, Pickard AS. Older medicare beneficiaries frequently continue medications with limited benefit following hospice admission. J Gen Intern Med. 2019. https://doi.org/10.1007/ s11606-019-05152-x.

2. Sera L, McPherson ML, Holmes HM. Commonly prescribed medication in a population of hospice patients. Am J Hosp Palliat Care. 2014; 31(2):12631.

3. Reimer C., et al. Proton-pump inhibitor therapy induces acid-related symptoms in healthy volunteers after withdrawal of therapy. Gastroenterology. 2009; 137(1):80-87.

Publisher's Note Springer Nature remains neutral with regard to jurisdictional claims in published maps and institutional affiliations. 